\title{
Filters Ranking for DWT-Domain Robust Digital Watermarking
}

\author{
Martin Dietze \\ Department of Information Systems, University of Buckingham, Buckingham MK18 1EG, UK \\ Email:martin.dietze@buckingham.ac.uk \\ Sabah Jassim \\ Department of Information Systems, University of Buckingham, Buckingham MK18 1EG, UK \\ Email: sabah.jassim@buckingham.ac.uk
}

Received 30 March 2003; Revised 24 September 2003

\begin{abstract}
In recent years a number of wavelet-based watermarking schemes have been proposed and exhibited improved qualities. The choice of a wavelet filter bank for a digital watermarking scheme can have a significant influence on the scheme's performance in terms of image quality and robustness. We present the results of experiments conducted using two different embedding algorithms (one blind and one nonblind) using a number of popular filter banks. The aim is to find filters that exhibit optimal performance with respect to specified requirements. The results demonstrate that the subband depth of embedding has the most significant influence on the filter bank choice. The kind of attack and the kind of embedding are also important, while marking intensity and compression ratio seem to affect the performance to a less extent. Additionally we show that out of the two embedding methods the quantization-based blind one leads to better overall results than the popular, nonblind one.
\end{abstract}

Keywords and phrases: watermarking, embedding, SCS, spread spectrum, wavelet, filters.

\section{INTRODUCTION}

Robust digital watermarking (e.g., for copyright protection) has gained increasing importance with the availability and popularity of Internet and eCommerce applications. Digital object formats do not restrict copying or further distribution of image files. Watermarking is used to assert rightful ownership or track down pirate copies by previous invisible embedding of a logo or a serial number into the file. The performance of watermarking schemes is measured in terms of two rather contradicting requirements: imperceptibility (i.e., optimally minimum image degradation) and robustness (i.e., withstanding various attacks that aim to remove the watermark or render it undetectable). Benchmarking tools like StirMark [1] combine most attacks and show that most existing watermarking schemes are vulnerable.

The advantages of DWT-based watermarking are wellaccepted, still apart from our own work [2] little is said in the literature about how the choice of a filter bank affects a watermarking scheme's performance. In [3], Fei et al. discuss the choice of a transform domain for watermarking, and in [4], Wolfgang et al. look at the effect of matching the domain of marking to the domain for lossy compression, yet both papers do not discuss the effect of a chosen domain's individual parameters.
Besides the choice of a filter bank, a DWT marking scheme's performance depends on features, like subband depth and the decomposition scheme used. Characteristics shared with nonwavelet-based schemes are the embedding technique and embedding intensity. Due to the DWT's spatial support, variation in texture, details, and gray scale/color are likely to have some impact too.

Recent watermarking schemes use a variety of different measures to achieve robustness. Most such schemes (see [5]) have a number of things in common: significant wavelet coefficients are chosen for embedding, information is embedded in single coefficients (normally through additive/multiplicative embedding), and often both blind or nonblind embedding are possible, resulting in different levels of robustness.

Different marking schemes may differ in the exact choice of coefficients for embedding, the algorithm that locates embedded mark, the intensity of embedding, the nature of the watermark (statistically undetectable, kind of message, etc.), and the detection device and decision process. Some schemes are designed to perform specific measures against certain attacks.

In this paper, we extend the work reported in [2], and compare the performance of some well-known filters 
in terms of image degradation resulting from embedding and the watermark quality after attacks. Locating previously marked coefficients does not really depend on the chosen filter bank, we thus focus on how particular filters cope with changes to marked coefficients.

For our experiments, we implemented a very simple watermarking scheme based on the common features listed above, which also allows to choose from two alternative embedding algorithms. We use 7 quality levels of JPEG compression and, to test for dependencies between the results and the kind of compression, a DWT-based compression at 4 different ratios as an attack on 8 different images. We then compare the results achieved with different filters to obtain general rankings.

Our results indicate that though there is no one optimal filter bank, good compromise choices can be found that can even be further optimized through additional measures. Also, one of the used embedding algorithms clearly outperforms the other more popular one.

The rest of the paper is organized as follows: in Section 2 we describe the watermarking system, the test course, and the tested filters and images. We then present and discuss our results in Sections 3 and 4. Appendix A contains the filter rankings and further information.

\section{EXPERIMENTAL SETUP}

\subsection{The watermarking system}

Our watermarking scheme shares the most commonly used features listed above and allows choosing between two different embedding techniques. For marking, the image is first decomposed into the DWT domain by a given number of steps using the Pyramid decomposition and a given filter bank. Assuming that the watermark is of size $n$, the $n$ most significant coefficients are picked from appropriate chosen marking subbands. For simplicity we store the marked coefficients' positions as part of the key needed for extracting the mark since the significant coefficients are likely to change position after marking. For embedding a mark bit $m_{i}$ in an image coefficient $x_{i}$ with a given intensity, we use two alternative algorithms - a nonblind and a blind one - that are explained in more detail below. After embedding, the image is reconstructed by using the inverse transform, and values outside the range [0-255] will be truncated to fit into it. We use the mean square error (MSE) to measure marking image degradation.

For detection, the marked image is decomposed, and the previously stored information on the embedding positions and-if applicable-original values are used to extract the mark. The embedded watermark is a binary image. Thus only ones and zeroes are embedded. The choice of such a watermark was inspired by [6]. For deciding about the presence of a mark, both, the human eye and an automatic detection device can be used to compare the detected with the original watermark. Because we mark coefficients sorted by their absolute values, it is easy to see which of them suffered worst from an attack (Figure 1).
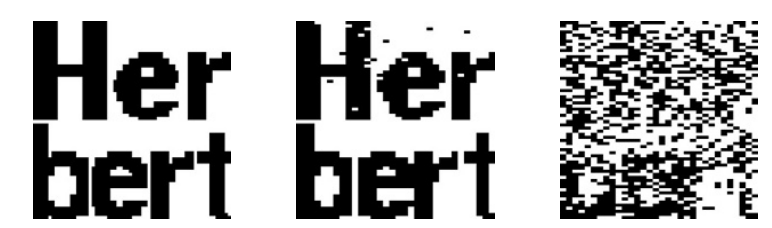

FigURE 1: The watermark before and after attacks.

\subsection{The nonblind embedding algorithm}

The nonblind embedding method is similar to the popular DCT-based spread spectrum scheme proposed in [7] (throughout this paper, $x_{i}$ denotes coefficients before and $y_{i}$ after marking, $m_{i}$ mark bits, and $x_{i}^{\prime}, y_{i}^{\prime}, m_{i}^{\prime}$ values obtained from a possibly attacked image):

$$
y_{i}=x_{i}\left(1+\alpha m_{i}\right)
$$

Embedding intensity is controlled by $\alpha$. To extract the watermark, the original values are needed:

$$
m_{i}^{\prime}=\frac{y_{i}^{\prime}-x_{i}}{\alpha x_{i}} .
$$

This multiplicative algorithm keeps the modification/values ratio constant, and its nonblind detection is independent from the host data.

For our experiments, we marked images using subband depths of 1,2 , and 3 and intensities ranging from $20 \%-80 \%$ on each of those subband depths.

\subsection{The blind embedding algorithm}

An obvious and a more practical choice of a blind scheme would be a variant of the above spread spectrum method. However, the detection scheme does not effect the measures under consideration (i.e., robustness and image degradation). Therefore, we chose an algorithm that changes coefficients in a way that differs from that done by the spread spectrum one.

The SCS embedding method was proposed by Eggers et al. [8] for the spatial domain. We use it for marking DWT coefficients and thus call it DWT-SCS:

$$
y_{i}=x_{i}+\alpha\left(\mathcal{Q}_{\Delta}\left\{x_{i}-\Delta\left(\frac{d_{i}}{2}+k_{i}\right)\right\}-x_{i}+\Delta\left(\frac{d_{i}}{2}+k_{i}\right)\right) .
$$

The system uses a linear quantizer $\mathcal{Q}$ with the stepwidth $\Delta$. The key $k$ is a pseudorandom sequence with $k_{i} \in(0,1]$. The embedding intensity is controlled mainly by the $\Delta$ parameter while $\alpha$ can be used to control the tradeoff between maximizing robustness by placing the mark near the center of a quantization cell and keeping the image distortion low. Since we are embedding binary sequences, the codeword $d_{i}$ is taken from the alphabet $\mathcal{D}=\{0,1\}$. For extraction, the pseudorandom sequence $k$ and the step width $\Delta$ are needed:

$$
m_{i}^{\prime}=\mathcal{Q}_{\Delta}\left\{y_{i}^{\prime}-\Delta k_{i}\right\}-\left(y_{i}^{\prime}-\Delta k_{i}\right) .
$$


Although detection is again independent of the host data, SCS does not keep the modfication/values ratios constant. It results in limited absolute changes for any combinations of $\Delta$ and $\alpha$.

We marked images using subband depths of 1, 2, and 3, each with 13 different intensity settings with $\Delta$ ranging from 20.0 to 120.0 . Due to the different ways these settings change the DWT coefficients, comparing the results from the multiplicative and the DWT-SCS embedding is not straightforward. Instead of the normalised rankings, we thus use the robustness/image quality tradeoff.

\subsection{The $L^{q_{d}}$ quality measurement}

To measure the watermark quality, we implemented a simple tolerant image comparison tool using a measure we call $L^{q_{d}}$, derived from the $L^{q}$ pseudonorm introduced by Jacobs et al. [9]. The $L^{q}$ measure is an image querying metric that uses truncated quantized versions of the wavelet decomposition and contains only the most significant information content of an image. The $L^{q}$ distance between two images essentially measures the differences in their most significant wavelet coefficients. For two images $I_{1}$ and $I_{2}$, fully decompose, select a fixed percentage of their most significant coefficients, set those values to 1 or -1 (depending on their original signs) and the rest to 0 , and the sum of weighted differences between the corresponding nonzero pixel values in $I_{1}$ and $I_{2}$ is the $L_{q}\left(I_{1}, I_{2}\right)$. The weights are subband dependent. Our $L^{q_{d}}$ differs from the $L^{q}$ in discarding the images' average (i.e., $L L)$ component. Also, since the summing up of the scores is asymmetric, the $L^{q_{d}}$ uses the lower of both possible values multiplied by a normalization factor as the result (see [2] for more details).

Measuring how well the mark is recognizable rather than the amount of distortion the $L^{q_{d}}$ mimics the way a human would try to read the watermark. This makes it a better choice than, for example, the MSE for the purpose of measuring a detected watermark's quality. We use experimentally obtained threshold categories for the watermark detection quality: clearly $(<21)$, still $(<25)$, partly $(<31)$, or only in traces $(<34)$ detectable.

\subsection{The filters and images used}

The filters used (coefficients taken from Davis' wavelet coding kit [10]) are given in Table 1.

The 8 images used in our tests are all gray-scale pictures of $512 \times 512$ pixel size. Most are well known in the watermarking community and in the public domain. They are available at our project pages in the Internet (http://herbert.the-little-red-haired-girl.org/en/research/ papers/filter_evaluation).

\section{RESULTS AND DISCUSSION}

The image degradation and watermark quality were tested against the following parameters: choice of filter, watermark intensity, (lossy) compression ratio, decomposition scheme, the chosen subband depth for marking, and the image itself. As the optimal choice of filter was our primary interest,
TABLE 1

\begin{tabular}{c|cc}
\hline Name & Orthogonality & Length \\
\hline Haar & Orthogonal & 2 \\
Daub4 & Orthogonal & 4 \\
Daub6 & Orthogonal & 6 \\
Daub8 & Orthogonal & 8 \\
\hline Antonini & Biorthogonal & $9 / 7$ \\
Odegard & Biorthogonal & $9 / 7$ \\
Villa2 & Biorthogonal & $13 / 11$ \\
Villa3 & Biorthogonal & $6 / 10$ \\
Villa4 & Biorthogonal & $5 / 3$ \\
Villa5 & Biorthogonal & $2 / 6$ \\
Villa6 & Biorthogonal & $9 / 3$ \\
\hline
\end{tabular}

we needed to look at all possible combinations of the other four parameters. Some of these are controllable within the scheme, others, like the image and the kind of attack, are beyond one's control in real-life watermarking. Also the filters' performance was found to depend only on some of them in a way that changing a parameter results in different rankings.

Our results reveal that the most significant correspondences between particular parameters and a filter's ranking are the subband depth for marking, the embedding method, and the kind of compression attack. The embedding intensity and the compression ratio made only little difference. This observation is of significant interest to all wavelet-based watermarking schemes. Consequently we grouped the ranking results by these three most significant parameters. The full rankings averaged over all tested images are shown in Appendix A.

\subsection{Image degradation}

Image degradation caused by watermarking was found to depend mostly on the subband depth allowed for marking. Marking only the first subband has little effect on the image, but increasing the embedding depth to the second subband can already lead to visible artifacts even at low embedding intensities on highly textured images like Barbara depending on the embedding method.

The first subband has relatively low energy, the choice of the most significant coefficients for marking thus moves most of the watermark towards the coarser scales.

In fact, subband 1 accommodates less than half of the watermark when subbands 1 and 2 are marked, and the ratio gets smaller if more subbands are marked. This can be explained by Figure 2 which shows the histogram of the wavelet coefficients in subbands 1 , and 1 and 2 together. Though similar in shape, the histogram for subband 1 alone (drawn with a thicker line) is much narrower.

Since every coefficient in subband $s$ corresponds to four coefficients in the next finer scale $s-1$, this leads to larger regions affected by changes through marking in coarser scales and thus potentially more image degradation. Depending on the length of the wavelet filter bank, the marking of the chosen DWT coefficients will have an effect on a number of pixels in the reconstructed image. A good filter with respect to 


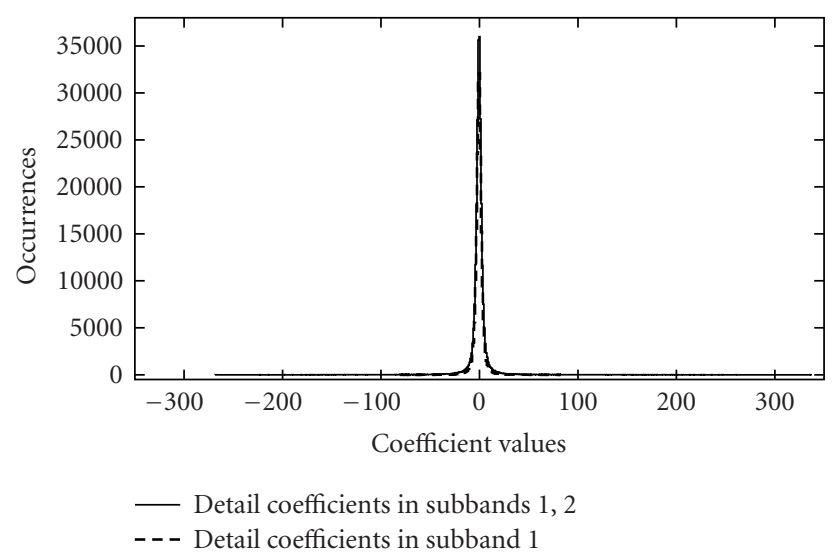

FIgURe 2: Coefficient values histogram of image Lena 512, decomposed using Villa 3; subbands 1-2.

image quality will "tolerate" this, so that visible artifacts in the reconstructed image are minimized. This is essentially the same with wavelet-based lossy compression. However, such compression will most likely affect different, more uniformly distributed coefficients and apply less significant changes. We thus expect filters with good properties for lossy compression to be potentially good choices for embedding where image quality is important. This explains some of the results below.

\subsubsection{Multiplicative embedding}

When using multiplicative embedding, biorthogonal filters, most of which are designed (like some of the Villa filters from [11]) or commonly used for compression (like Antonini from [12]) exhibit best performances. On the other hand, the orthogonal filters Haar, Daub4, Daub6, and Daub8 are on the bottom of the rankings.

Some filters (e.g., Villa4, Villa6) perform well only at low and others (Antonini, Villa5) at high subband depth, but there is also an excellent all-rounder (Villa3).

The subband depth of marking has no significant influence on ranking distances between the filters. The spread found between the best and the worst filter slightly decreases from 1.4 (subband depth 1) to 1.3 (subband depth 2, 3) and is more or less evenly distributed.

\subsubsection{DWT-SCS embedding}

The DWT-SCS embedding leads to different rankings. First, a group of three filters lead the ranking at subband depths of 2 and 3: Villa6, Villa4, and Villa3. Interestingly this group is at the bottom at subband depth 1 . The four orthogonal filters form a close group in all degradation ranking, and perform much better than when used with the multiplicative method. They are on top at subband depth 1 and just after the top group at depths 2 and 3 .

At subband depth 1, the results need a closer look, since the differences between the filters are very small. However unlike the multiplicative method, at higher subband depth we get a significant spread within each ranking table growing with increased subband depth (approaching 2 at depth 3 ).
Interestingly increasing the subband depth does not lead to increased image distortion with all filters. This stands in contrast to the multiplicative embedding, but is quite logical since the uniform quantization used in the DWT-SCS embedding leads to higher relative changes to coefficients with lower absolute values. As the proportion of coefficients with high absolute values increases with subband depth, a higher subband depth causes more subtle changes to the marked coefficients' values. But the one to four correspondence between coefficients in successive subbands means that changes to coefficients in coarser subbands lead to potentially more image degradation.

The results obtained using the DWT-SCS embedding method show that the balance between these two factors depends a lot on the filter used for embedding. Actually only with Villa5, Odegard, and Villa2, which are also found at the bottom of the rankings at higher subband depths, the image degradation increases with the subband depth of marking. These observations remain the same regardless of the intensity settings used.

\subsubsection{Overall degradation results}

With both embedding algorithms, the subband depths of marking 2 and 3 lead to relatively consistent rankings differing slightly from the ones obtained at subband depth 1 . The results are different at a subband depth of 1 , however the filters' results with the DWT-SCS embedding are so close to each other that we may not want to overestimate this observation. Interestingly there are some filters with almost similar performance at the subband depths 2 and 3 with both embedding techniques_-Villa3 and to a lesser extent Villa4 at the top, and Villa2 at the bottom. The popular orthogonal filters (Haar, Daub 4, 6, and 8) seem recommendable only for the DWT-SCS, and Antonini only for the multiplicative embedding algorithm, respectively.

\subsection{Watermark quality}

Our results show that our two embedding techniques differ a lot with respect to the watermarking software's performance but do not seem to influence the rankings. In this section, we first discuss the differences found between the results from using the two different embedding techniques. After that, we look at the actual filter rankings in terms of the type of compression attack.

\subsubsection{Multiplicative embedding}

Similar to the degradation rankings (see Section 3.1) progression of the scores associated with the differently ranked filters is relatively stable with the multiplicative embedding. Regardless of the filter bank, a watermark inserted in the first subband needs intensities of more than 30\% to survive JPEG compression with quality factors less than 95\%. However, marking only the first two subbands dramatically improves the detection results. Even with low embedding intensity of $20 \%$ the mark is still clearly detectable at JPEG quality factors of $85 \%$ or more. Marking the first three subbands or even more makes the watermark virtually invulnerable to lossy compression. 
This can be explained by the multiplicative embedding formula, $y_{i}=x_{i}\left(1+\alpha m_{i}\right)$-marking large coefficients leads to high absolute changes. A compression attack using a uniform quantizer will thus need a high quantization step to destroy the watermark which is impractical because of the resulting poor image quality. Even nonuniform quantizers would have to operate in the same DWT domain as the one chosen for embedding the mark to attack a mark embedded in the most significant coefficients more efficiently. Since, as mentioned in Section 3.1, the coarser subbands 2 and 3 usually contain higher proportion of significant coefficients, allowing more subbands for marking results in a dramatic increase of the robustness of the watermark at the cost of image quality.

\subsubsection{DWT-SCS embedding}

The blind DWT-SCS embedding produced overall good results which were not worse than the nonblind multiplicative one's. At subband depth 1 both methods exhibit the same performance at a given image degradation. In contrast to the observation made with the multiplicative embedding technique, an increased subband depth of marking does not automatically lead to a more robust watermark. However as the level of degradation caused by marking goes down at higher subband depths (Section 3.1.2), higher values of $\Delta$ can be selected for the watermark intensity which more than compensates the missing gain of robustness; actually the product between image and watermark degradation at subband depths 2 and 3 is usually lower than the corresponding one we get with the multiplicative method.

To find possible reasons for this behaviour, we can use almost the same considerations as for the image degradation (see Section 3.1.2). The DWT-SCS's uniform quantization leads to relatively large changes to coefficients with low and little changes to those with large values. Whether or not an increased subband depth leads to improved robustness, depends most of all on the kind of attack: the more similar the attack's kind of quantization is to the one used for embedding, the less an increased subband depth of marking (at the same intensity level) will lead to improved robustness. In our experiments, we found hardly any difference with the DWT-based and only slight robustness improvements with the JPEG compression attack. Since the selection of coefficients and the uniform quantization used by the DWT-based compression is more similar to our embedding in the DWTdomain than the (DCT-based) JPEG compression, this result supports our above statement.

Similar to the results from the degradation rankings, the progression within the rankings is rather high at higher subbands while there is hardly any difference between the filters if only the first subband is marked.

\subsubsection{JPEG compression rankings}

The group of orthogonal filters show the best robustness against JPEG compression. In the image quality rankings, the same group is ranked in the midfield with some distance to the top group when using DWT-SCS, and even at the bottom when using the multiplicative embedding, so obviously these filters are the best choices for watermarking optimized on robustness. Interestingly, we find one of the biorthogonal filters, Villa3, consistently ranked in the top group. This result is remarkable because this filter bank had already shown very good properties with respect to image quality. In our experiments no other filter had a comparable all round capability. In the bottom group of the tables we consistently find Villa4 that had been in the top group of all image degradation rankings. The rest of the filter banks in the lower half of the tables differ depending on the subbands depth of marking.

\subsubsection{DWT-based compression rankings}

We tested the filters against DWT-based compression at only four different quality factors to find out how a different kind of compression affects the results. For the attack we used Davis' simple transform coder [10] which uses quantization and entropy coding in the DWT domain and the Antonini filter for decomposition. The rankings are quite different here.

The orthogonal filters were not in the top group anymore, while the biorthogonal ones with long sets of coefficients provided the best robustness. Interestingly, the best detection results were achieved with filters of nearly the same length of the Antonini filter used for the compression attack. Additional tests (see [2] for more details) revealed that the rankings of the filters used for embedding are significantlybut not in an obvious way-influenced by the choice of filter for the compression attack. Similar observations were made when repeating these experiments using DWT-SCS embedding. This effect was in a way suggested by [4] in which similar transform domains for marking and compression were found to be beneficial for detection. Further investigation of this correspondence, and the design of a filter bank with good robustness against any kind of DWT-based compression could be the starting point for interesting followup research. In general, long filters give the best results, but the actual rankings change with the choice of filter for compression and with other parameters, so that no simple recommendation can be made here.

\subsubsection{Overall detection results}

Regardless of the embedding method, both rankings depend most of all on the subband depth of marking and the kind of compression attack used after marking. Unfortunately two kinds of compression attacks suggest using different filters, so a filter will either optimize the scheme against one attack or be a compromise. Such a compromise could be Daub8 with its very good robustness against JPEG and, since it is a long filter bank, reasonably good robustness against DWT compression.

\subsection{Overall results}

In most situations, the DWT-SCS has overall properties superior to the multiplicative method.

Marking an image with the two methods to approximately similar level of image degradation, the multiplicative method was only found to have better overall results than the 
SCS at subband depth 1 and a low compression quality factor used for the attack. In all other cases, especially at higher subband depths, the DWT-SCS clearly outperforms the multiplicative technique.

\subsection{Possible optimizations}

Imperceptibility and robustness usually have conflicting requirements. To achieve the best possible robustness at a given level of image degradation, additional fine tuning is necessary. Since the two adopted embedding methods differ significantly in this respect we need to look at possible optimized settings separately.

\subsubsection{Tuning multiplicative embedding}

Good robustness is only achieved when marking more than only the first subband, but this easily results in visible artifacts in the marked image. Because of the large absolute changes to large coefficients (Section 3.2.1), robustness quickly goes into saturation at high subband depths. We can thus scale down the intensity in higher subbands (as proposed in [13]) for a better tradeoff between image quality and watermark robustness.

\subsubsection{Tuning DWT-SCS embedding}

The quantization-based embedding does not provide a similar simple dependency between its intensity settings and performance. In fact, adapting the $\Delta$ values to the marked subband introduces a somewhat random effect on the results. While upscaling leads to proportionally increased image degradation, the detection results do not exhibit a similar kind of behaviour. There is an obvious interaction between the quantization performed by the embedding process and the one resulting from the attacking compression ratio.

The optimal settings thus depend on the weight put on image quality and robustness. Since only the image quality is usually under one's control, the optimization process starts with selecting an upper bound on allowed image degradation. Settings (intensity, choice of filter) leading to not more than the upper bound can be experimentally determined, and the selected combination should provide the best possible robustness against a given attack. However this optimization does not seem as vital as with the multiplicative embedding, because DWT-SCS usually provides reasonable performance at nonadaptive settings.

\section{CONCLUSIONS, FUTURE WORK}

We investigated parameters that influence the best choice of filter banks with the aim of finding a good compromise between the competing requirements of imperceptivity and robustness. We have established that for both requirements a filter's performance depends most of all on the subband depth used for marking. While finding good filters for optimal image quality is easy, the detection results are influenced by the kind of attack which is beyond our control in real-life watermarking.
To achieve good robustness against JPEG compression allowing little image degradation, the Villa3 filter bank is more than a compromise if the first two or three subbands are chosen for embedding. However, the DWT-based compression attack leading to different results suggests that the kind of (compression) attack has an impact on the optimal choice of filter bank, too. Because the way an attack is conducted is beyond one's control in real life, no one filter can be recommended as an optimal choice here, but in general, long filters showed good results. While Villa3 has good overall properties regardless of the two embedding techniques we tested, Antonini is a relatively good choice if the multiplicative one is used, and Daub8 if the DWT-SCS embedding is used. From the two different embedding techniques we used in our experiments, the blind DWT-SCS clearly outperformed the nonblind multiplicative embedding.

For robustness to the two tested attacks, marking more than the first subband is desirable, but it easily leads to artifacts. Depending on the embedding algorithm adaptive marking with changing intensity settings depending on the marked coefficients' subbands can help in achieving better overall results.

This work is part of an ongoing project on wavelet-based second generation watermarking (see [14]). Such schemes try to increase robustness against geometric distortion attacks (e.g., StirMark [1]). The need for such advanced techniques gets obvious once we take more sophisticated attacks than lossy compression into consideration. For example, watermark embedded using a Villa3 filter and DWT-SCS embedding with $\Delta=40.0$ and $\alpha=1.0$ at subband depth 2, easily survives JPEG compression of a quality factor of $65 \%$ and DWT compression at a compression ratio of $1: 14$, but was rendered completely unreadable after a StirMark [1] allowing only geometric attacks at standard settings.

Our next step will be to extend our marking system for selecting and locating marked coefficients according to features in the DWT-transformed image.

\section{APPENDICES}

\section{A. THE RANKINGS}

Here, we introduce the full rankings averaged overall tested images. In Tables 2, 3, 4, 5, 6, and 7, the abbreviations used are Ha (Haar), D4 (Daub4), D6 (Daub6), D8 (Daub8), An (Antonini), Od (Odegard), V2 (Villa2), V3 (Villa3), V4 (Villa4), V5 (Villa5), and V6 (Villa6).

\section{B. DEGRADATION/DETECTION EXAMPLES}

In Figure 3, the corresponding MSEs resulting from marking are 15.92560 (M70) and 2.09668 (S40). In contrast to M70, S40 causes hardly any perceptible image degradation.

The detection results in Figure 4 were scored in $L^{q_{d}}$ as 3.56445 (M70) and 6.10352 (S40). Both detection results are thus well below the experimentally determined readability thresholds (see Section 2.4 for the actual thresholds).

All in all S40 exhibits superior overall performance. 
TABLE 2: Detection rankings (multiplicative embedding) after JPEG compression $\left(L^{q_{d}}\right)$ (Sections 3.2.1, 3.2.3).

\begin{tabular}{lrlrlr}
\hline \multicolumn{2}{c}{ 1 subband } & \multicolumn{2}{c}{ 2 subbands } & \multicolumn{2}{c}{3 subbands } \\
\hline Ha & 16.453 & D8 & 10.259 & V3 & 8.715 \\
D4 & 17.704 & V3 & 10.533 & D4 & 9.057 \\
D6 & 17.725 & D6 & 10.678 & D6 & 9.059 \\
D8 & 17.894 & Ha & 10.807 & D8 & 9.133 \\
V5 & 18.562 & D4 & 11.016 & Ha & 9.260 \\
V2 & 19.169 & Od & 11.529 & V6 & 9.337 \\
Od & 19.703 & V6 & 11.660 & An & 9.415 \\
An & 19.727 & V5 & 11.969 & V5 & 9.788 \\
V3 & 19.778 & An & 12.268 & V2 & 9.911 \\
V6 & 20.621 & V2 & 12.327 & V4 & 9.933 \\
V4 & 20.895 & V4 & 12.467 & Od & 10.098 \\
\hline
\end{tabular}

TABLE 3: Detection rankings (multiplicative embedding) after DWT compression $\left(L^{q_{d}}\right)$ (Sections 3.2.1, 3.2.4).

\begin{tabular}{llllll}
\hline \multicolumn{2}{c}{ 1 subband } & \multicolumn{2}{c}{ 2 subbands } & \multicolumn{2}{c}{3 subbands } \\
\hline An & 19.269 & Od & 11.645 & V2 & 10.053 \\
Od & 19.698 & An & 12.012 & An & 10.170 \\
V2 & 19.750 & V6 & 12.263 & V6 & 10.288 \\
V4 & 21.041 & V2 & 12.385 & Od & 10.340 \\
V6 & 21.275 & V4 & 12.666 & V4 & 11.733 \\
Ha & 24.483 & D8 & 20.992 & D8 & 15.469 \\
V5 & 26.803 & Ha & 21.019 & Ha & 17.135 \\
V3 & 27.648 & V5 & 22.862 & D6 & 18.107 \\
D8 & 27.934 & V3 & 24.663 & V5 & 18.728 \\
D4 & 29.471 & D6 & 25.014 & V3 & 19.364 \\
D6 & 30.346 & D4 & 25.223 & D4 & 19.733 \\
\hline
\end{tabular}

TABLE 4: Detection rankings (DWT-SCS embedding) after JPEG compression $\left(L^{q_{d}}\right)$ (Sections 3.2.2, 3.2.3).

\begin{tabular}{llllll}
\hline \multicolumn{2}{c}{ 1 subband } & \multicolumn{2}{c}{ 2 subbands } & \multicolumn{2}{c}{ 3 subbands } \\
\hline Ha & 6.035 & D8 & 3.018 & D8 & 1.796 \\
D6 & 6.114 & D4 & 3.115 & D4 & 1.933 \\
V3 & 6.160 & D6 & 3.146 & D6 & 1.935 \\
D8 & 6.232 & V3 & 3.167 & V3 & 2.123 \\
D4 & 6.324 & Ha & 3.430 & Ha & 2.267 \\
V5 & 6.471 & Od & 3.512 & V5 & 2.397 \\
V2 & 6.847 & V2 & 3.605 & Od & 2.408 \\
V6 & 6.891 & V5 & 3.669 & V2 & 2.440 \\
An & 6.939 & An & 3.833 & An & 2.545 \\
Od & 6.949 & V6 & 4.403 & V6 & 2.883 \\
V4 & 7.005 & V4 & 5.183 & V4 & 3.827 \\
\hline
\end{tabular}

\section{SUMMARY ON THE TOOLS AND TEST PARAMETERS}

Our watermarking software was implemented on base of our $\mathrm{C}++$ Wavelet and Image class library which among others also includes a CLI program pgmcompare which we used to determine the MSE and $L^{q_{d}}$ scores for our experiments. The class library is Free Software and can be downloaded from
TABLE 5: Detection rankings (DWT-SCS embedding) after DWT compression $\left(L^{q_{d}}\right)$ (Sections 3.2.2, 3.2.4).

\begin{tabular}{lrlrlr}
\hline \multicolumn{2}{c}{ 1 subband } & \multicolumn{2}{c}{ 2 subbands } & \multicolumn{2}{c}{3 subbands } \\
\hline V4 & 7.298 & Od & 4.658 & Od & 4.002 \\
V6 & 7.424 & An & 4.847 & V2 & 4.032 \\
An & 7.450 & V2 & 4.873 & An & 4.076 \\
Od & 7.905 & V6 & 7.180 & V6 & 7.184 \\
V2 & 7.915 & V4 & 7.557 & D8 & 7.996 \\
V5 & 9.067 & Ha & 10.560 & V4 & 8.271 \\
V3 & 9.221 & D8 & 10.862 & V5 & 10.003 \\
Ha & 9.612 & V5 & 10.880 & Ha & 10.007 \\
D8 & 10.695 & D4 & 13.588 & D6 & 10.496 \\
D6 & 12.268 & D6 & 14.030 & D4 & 11.792 \\
D4 & 12.455 & V3 & 14.094 & V3 & 14.516 \\
\hline
\end{tabular}

TABLE 6: Degradation rankings multiplicative embedding (MSE) (Section 3.1.1).

\begin{tabular}{llllll}
\hline \multicolumn{2}{c}{ 1 subband } & \multicolumn{2}{c}{ 2 subbands } & \multicolumn{2}{c}{3 subbands } \\
\hline V4 & 5.326 & V4 & 12.919 & V3 & 26.494 \\
V6 & 5.464 & V3 & 13.118 & An & 27.358 \\
V3 & 5.534 & An & 13.313 & V5 & 27.468 \\
An & 5.573 & V6 & 13.340 & V4 & 27.543 \\
Od & 5.715 & V5 & 13.724 & Od & 27.937 \\
V2 & 5.867 & Od & 13.739 & V6 & 27.976 \\
V5 & 6.039 & V2 & 14.020 & V2 & 28.508 \\
D4 & 6.381 & D8 & 14.913 & D8 & 30.077 \\
D6 & 6.501 & D4 & 15.157 & D4 & 30.880 \\
D8 & 6.549 & D6 & 15.413 & D6 & 31.467 \\
Ha & 7.427 & Ha & 16.919 & Ha & 33.997 \\
\hline
\end{tabular}

TABLE 7: Degradation rankings DWT-SCS embedding (MSE) (Section 3.1.2).

\begin{tabular}{lclrlr}
\hline \multicolumn{2}{c}{ 1 subband } & \multicolumn{2}{c}{ 2 subbands } & \multicolumn{2}{c}{3 subbands } \\
\hline D4 & 13.939 & V6 & 10.152 & V6 & 8.495 \\
Ha & 13.947 & V4 & 10.573 & V4 & 8.919 \\
V2 & 14.071 & V3 & 11.096 & V3 & 9.341 \\
D6 & 14.084 & Ha & 14.019 & Ha & 13.809 \\
Od & 14.144 & D4 & 14.047 & D4 & 13.842 \\
D8 & 14.157 & D8 & 14.058 & D6 & 13.881 \\
An & 14.496 & D6 & 14.082 & D8 & 13.883 \\
V5 & 14.564 & An & 14.148 & An & 14.551 \\
V3 & 14.883 & V5 & 15.044 & V5 & 15.442 \\
V6 & 15.307 & Od & 15.526 & Od & 17.068 \\
V4 & 15.445 & V2 & 16.243 & V2 & 18.145 \\
\hline
\end{tabular}

http://herbert.the-little-red-haired-girl.org/en/research/index. html\#software.

We used the CLI tools cjpeg/djpeg (JPEG distribution, http://www.ijg.org/files), and encode/decode (Davis' wavelet kit [10]) to perform the two kinds of lossy compression.

For the JPEG attack, cjpeg was called in the following way: "cjpeg-quality quality -outfile tmp.jpg image" with the quality factors $100,95,90,85,80,70$, and 60 . For the DWT 


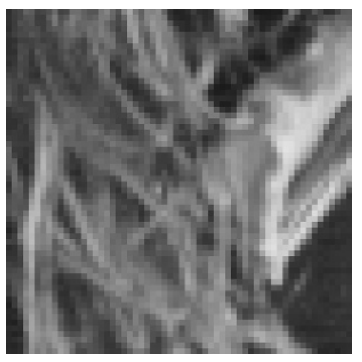

(a)

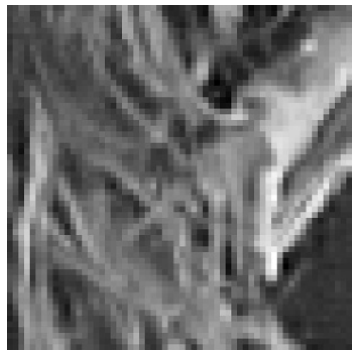

(b)

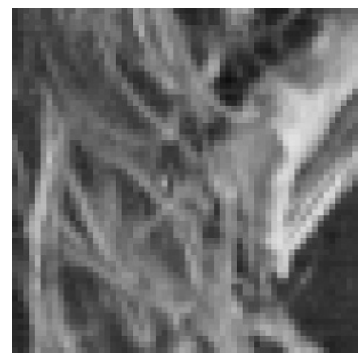

(c)

Figure 3: (a) Original, (b) Daub4 multiplicative at 70\% (M70), and (c) Daub4 DWT-SCS at $\Delta=40$ and $\alpha=1.0($ (S40).

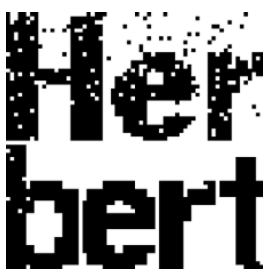

(a)

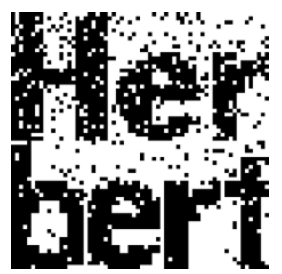

(b)
FIgURE 4: After JPEG (70\%): (a) M70 and (b) $S 40$.

attack, encode was called in the following way: "encode image tmp.raw ratio" with the ratio settings $8,10,12,14$, and 16 (for $1: 8,1: 10, \ldots$ ).

Throughout our experiments we used PGM images that were converted to other formats where necessary.

Our Unix shell scripts for automating the experiments are available upon request.

\section{REFERENCES}

[1] F. A. Petitcolas, R. J. Anderson, and M. G. Kuhn, "Attacks on copyright marking systems," in Proc. 2nd International Workshop on Information Hiding (IH '98), pp. 218-238, Portland, Ore, USA, April 1998, Introduces the StirMark benchmark software.

[2] M. Dietze and S. Jassim, "The choice of filter banks for wavelet-based robust digital watermarking," in Proc. Multimedia and Security Workshop at ACM Multimedia, pp. 37-41, French Riviera, France, December 2002.

[3] C. Fei, D. Kundur, and R. Kwong, "The choice of watermark domain in the presence of compression," in Proc. IEEE International Conference on Information Technology: Coding and Computing (ITCC '01), pp. 79-84, Las Vegas, Nev, USA, April 2001.

[4] R. Wolfgang, C. Podilchuk, and E. Delp, "The effect of matching watermark and compression transforms in compressed color images," in Proc. IEEE International Conference on Image Processing (ICIP '98), vol. 1, pp. 440-444, Chicago, Ill, USA, October 1998.

[5] P. Meerwald, "Digital image watermarking in the wavelet transform domain," M.S. thesis, Department of Scientific Computing, University of Salzburg, Salzburg, Austria, 2001.

[6] W. Zeng, B. Liu, and S. Lei, "Extraction of multiresolution watermark images for resolving rightful ownership," in Secu- rity and Watermarking of Multimedia Contents, vol. 3657, pp. 404-414, San Jose, Calif, USA, January 1999.

[7] I. J. Cox, J. Kilian, T. Leighton, and T. Shamoon, "Secure spread spectrum watermarking for multimedia," IEEE Trans. Image Processing, vol. 6, no. 12, pp. 1673-1687, 1997.

[8] J. J. Eggers, J. k. Su, and B. Girod, "A blind watermarking scheme based on structured codebooks," in IEE Colloquium: Secure Images and Image Authentication, pp. 4/1-4/21, London, UK, April 2000.

[9] C. E. Jacobs, A. Finkelstein, and D. H. Salesin, "Fast multiresolution image querying," Computer Graphics, vol. 29, pp. 277-286, November 1995.

[10] G. Davis, "Wavelet Image Compression Construction Kit," January 1997, http:/www.geoffdavis.net/dartmouth/wavelet/ wavelet.html.

[11] J. D. Villasenor, B. Belzer, and J. Liao, "Wavelet filter evaluation for image compression," IEEE Trans. Image Processing, vol. 4, no. 8, pp. 1053-1060, 1995.

[12] M. Antonini, M. Barlaud, P. Mathieu, and I. Daubechies, "Image coding using wavelet transform," IEEE Trans. Image Processing, vol. 1, no. 2, pp. 205-220, 1992.

[13] J. R. Kim and Y. S. Moon, "A robust wavelet-based digital watermarking using level-adaptive thresholding," in Proc. IEEE International Conference on Image Processing (ICIP '99), vol. 2, pp. 226-230, Kobe, Japan, October 1999.

[14] M. Kutter, S. Bhattacharjee, and T. Ebrahimi, "Towards second generation watermarking schemes," in Proc. 6th IEEE International Conference on Image Processing (ICIP '99), vol. 1, pp. 320-323, Kobe, Japan, October 1999.

Martin Dietze is a graduate of the University of Applied Sciences, Wedel, Germany, with the degree "Diplomingenieur der Technischen Informatik" (Engineer of Technical Computer Science) in 1997. While still a student, he worked for IBM and some smaller software companies. After receiving his degree he stayed at the University of Applied Sciences, Wedel, to work as a System Administrator Teaching Assistant

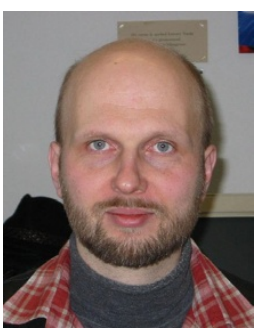
in software development, where he started working on his D.Phil. on second generation watermarking in the wavelet domain as a part-time project at the University of Buckingham in 1999. He then joined the University of Buckingham in 2001 to work as a Lecturer and to continue working on his D.Phil. Since 2003 Martin has been back in Germany, now working as a Research Associate in a project on repairing and texturizing polygon models for VR applications. He expects to finish his D.Phil. before the end of 2004. 
Sabah Jassim is a mathematics graduate of Baghdad University and holds a D.Phil. degree in algebraic topology which he received from the University of Wales-Swansea in 1980. Currently he is a Senior Lecturer in mathematics at the University of Buckingham, UK. He also holds visiting lecturing posts at City University, London, UK, and at the University of Applied Sciences, Wedel, Germany. Before joining the University of

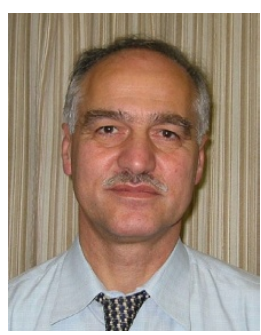
Buckingham, he lectured at the University College of Swansea, and Leicester Polytechnic, UK. His research interests and publications cover a wide range of mathematical applications in computing (e.g., information security, wavelet-based compression techniques for on-line transmission of medical video images, algebraically designed data structures for the computation of Delaunay triangulations and other geometric objects, and deadlock analysis of large-scale process networks). He is currently sponsored to research wavelet-based biometrics for face recognition. 